\title{
Early Assessment in Autism Spectrum Disorders
}

Thomas Layton ${ }^{1}$ and Grace $\mathrm{Hao}^{2 *}$

${ }^{1}$ Talk and Total Communication, Durham, NC, United States

${ }^{2}$ Communication Disorders Program, NCCU Durham, NC, United States

"Corresponding author: Grace Hao, Program of Communication Disorders, NCCU Durham, NC, United States, Tel: 919-530-7836, E-mail: jhao@nccu.edu

Received: June 18, 2014; Accepted: August 04, 2014; Published: August 25, 2014

Copyright: $\odot 2014$ Layton T et al., This is an open-access article distributed under the terms of the Creative Commons Attribution License, which permits unrestricted use, distribution, and reproduction in any medium, provided the original author and source are credited.

\begin{abstract}
Evaluating children with Autism Spectrum Disorders (ASD) is both an art and a science, and can be sometimes complicated. Due to the rapid increase of prevalence of ASD worldwide in the past decades, more professionals are being challenged to provide services to more children with ASD. Not only critical decisions have to be made to determine if a child is on the spectrum or not, but detailed evaluation procedures are also needed to determine the child's current functional level, which will serve as the guideline for treatment planning. The purpose of this article is to provide a suggested protocol to help professionals in the evaluation process. To begin with a brief background introduction of ASD is followed by the early development and characteristics of ASD, and it concludes with a detailed protocol of the early assessment areas and recommended diagnostic instruments. A good deal could be added to the discussion but it is hoped that this guide provides the basic understanding for professionals as to where to proceed in the evaluation of children with ASD
\end{abstract}

Keywords: Autism spectrum disorders; Domains; Assessment

\section{Introduction}

The Diagnostic and Statistical Manual-V (DSM-V) [1] uses the term "autism spectrum disorders" (ASD) as the umbrella term for children with Social/Communicative deficits and Restricted Interests/ Repetitive Behaviors. Social communication and social interaction includes behaviors such as failure to initiate or respond to social interactions [1]. It also includes deficits in nonverbal communication, poor eye contact, failure to understand gestures, and no comprehension of facial expressions. Social deficits include failure in developing, maintaining, and understanding relationships, such as, difficulties in adjusting to social contexts, difficulties in imaginative play or making friends, and absence of peers [1]. Restricted and repetitive patterns of behavior, interests, or activities include deficits such as stereotyped behaviors, insistence on sameness, highly restricted interests, hyper- or hypo-reactivity to sensory input [1].

Evaluation and treatment goals are based on identifying the above behaviors, as well as the severity levels related to the amount of support the child will need, rather than a simple diagnosis [1]. A child may be diagnosed as Level-3 where the child requires very supportive help in order to function: a Level-3 child has minimal communication and marked interference in daily life. A child at Level-2 severity requires substantial support, and the individual has limited initiations or atypical responses. A child at Level-1 severity requires some support, and the individual has some significant deficits in social communication, and at least one significant problem in restricted/ repetitive behaviors.

Diagnosing ASD, based on the DSM-V criteria, excludes language as an independent criteria; however, language assessment is encouraged as a related behavior to consider to help determine the level of severity. It is important to determine whether the child has intelligible speech and language. Does the child use single words, phrases, or complete sentences? Are the child's sentences fluent, monotone, or complex? Is there a gap in the child's receptive language (i.e. language comprehension skills) versus his/her expressive language?

The remainder of this paper covers the areas of early language development, available tests for measuring important areas and recommendations for a complete evaluation.

\section{Early Development of ASD}

The course of early development is an important indicator of whether the child presents with ASD or other non-ASD impairments. Early symptoms frequently occur during the second year of life but may be observed before 12 months of age [2]. It is also important to determine the early language skill of the child. Did the child have any words by age 12 months? How many words? Did the child loose these words? If so, how does the child communicate now? Tager-Flushberg [3] has reported two potential early courses of development in children with ASD. One course of development is the child gaining words consistently. The other course is the child had words and then lost them. Early language and oral vocabulary are good predictors of later language use among children with ASD [4]. Screening and evaluating children with ASD before age 2-years is recommended by the American Pediatric Association [5]. Delaying assessment until preschool or school age misses the opportunity for early intervention, such as establishing joint attention and imaginative play, important skills for social relationships, academic success, and independent living. Therefore, early measures of speech, language, social skills, and other risk factors for ASD are important.

Three frequently used early-language assessment measures are the Receptive-Expressive Emergent Language Test - Third Edition (REEL-3) [6], the Rossetti Infant-Toddler Language Scale (Rossetti) [7], and the MacArthur-Bates Communicative Development Inventories (MacArthur-Bates CDI) [8]. The REEL has two core subtests, Receptive Language and Expressive Language, and is useful for infants and toddlers. It takes approximately 20 minutes to 
administer. The Rossetti is a criterion-referenced instrument that measures Interaction-Attachment, Pragmatics, Gesture, Play, Language Comprehension, and Language Expression. It measures children from zero to 36 months of age. Both direct observation and parental reporting are utilized. Testing time varies but usually takes less than 20 minutes to complete. The MacArthur-Bates CDI contains three different components: CDI: Words and Gestures (for children 8 - 18 months), CDI: Words and Sentences (for children 16-30 months), and CDI-III (for children 30-37 months). The CDI: Words and Gestures assess comprehension and production vocabulary across various categories (i.e., toys, house items, foods, prepositions, etc.). The other two CDI components measure only production vocabulary. All three CDI components use parents as informants.

Currently, there are two commonly used early assessment instruments to assist in identifying children with ASD. The First Year Inventory and First Year Inventory, Lite (FYI, Lite) $[9,10]$ is a parent report to identify one-year old infants at risk for later developing ASD. Reznick et al. [10] examined the psychometric components of the FYI and found constructs within a social-communication and a sensoryregulatory domain. There are a total of 60 items included in the FYI. The newer version, FYI, Lite contains only 20 items [9]. The second instrument is the Autism Observation Scale for Infants (AOSI) [11,12]. The AOSI is an 18-item direct observation measure for infants from 6-18 months of age. The activities encourage an interactive context where the examiner plays with the infant while eliciting particular behaviors. Behaviors include visual tracking, orientation to name, imitation, eye contact, plus more.

\section{Related Areas}

Layton et al. [13] have found the early play-skills and social interaction are highly inter-related among young children with ASD. The children with limited imaginative play, restricted interests, and repetitive behaviors are also those children with limited eye contact, no pointing skills, fewer peer interaction, and less social exchanges. They are also those children with fewer friends and limited understanding of social rules, like turn-taking and dialoguing [14].

It is also important to assess the young child's oral-motor skills, feeding, and sensitivity to foods and textures. Many young children with ASD have issues with eating a variety of foods [15]. This is an important indicator for later speech development, since it has been documented that children with or without ASD, with poor chewing skills, inappropriate swallowing, and poor tongue-tip/lip closure, are candidates for poor speech sound production later on [16]. An assessment, therefore, should include observation of the child chewing, swallowing, and a parental report of food choices.

Children with sensory issues to food flavors, texture, and smells may also have sensory issues to touch, warmth/cold, soft/course, and loud sounds. These children may require a sensory-integration [17] approach to help them improve in their sensory profile. It has been found that children with sensory issues present with limited expressive vocabulary since they are reluctant to eat a variety of foods, touch a variety of objects, and react negatively to sounds and smells [17]. Their interests and vocabulary are limited. A thorough assessment of oralmotor skills, eating, and sensory concerns is recommended.

\section{Co-morbidity Disabilities}

It has been estimated that nearly 70 percent of children with ASD present with some other form of disability [1]. These related disabilities play an important role in the overall level of severity of the child, future progress, and targeted treatment goals needed. It is important, therefore, to include related disabilities in the assessment and evaluation of children with ASD.

One broad related area of disability is associated with known medical or genetic conditions; for instance, there are several known genetic disorders that present with ASD. These include, but are not limited to, Rett syndrome, Fragile-X syndrome, Down syndrome, and medical disorders like epilepsy. In addition, 10-15 percent of children with ASD have been identified with a single-gene or chromosome abnormality [18].

Environmental conditions of ASD are also common factors that play a role within this population [19]. Such causes may be prenatal conditions, or early environmental exposure to adverse conditions, e.g. high fevers, measles, lead poisoning, and other toxic conditions. Prenatal conditions may include valproate exposure, fetal alcohol syndrome, and very low birth weight. Anything that may have a lasting effect on the child's development needs to be reported.

Additional neurodevelopmental, mental, or behavioral conditions also need to be reported. These may include Attention Deficit Hyperactivity Disorder (ADHD), disruptive behavior, and impulsecontrol or conduct behavior, anxiety, depression, bipolar disorders, tics or Tourette's syndrome, self-injury behavior, and sleep disorders.

Intellectual disability is a critical area of assessment [20]. It is a preferred practice to administer a standardized test of intelligence along with a diagnostic test for ASD. A child with intellectual disabilities without ASD is hard to differentiate from a young child with ASD because both may have language impairments and social communication issues. When social communication and interaction are significantly impaired relative to the child's non-verbal skills then a diagnosis of ASD is appropriate and recommended.

\section{Screening for ASD}

Screening for a disorder should never replace a diagnostic test [21]. A first-level screening is when the test is administered on a large number of individuals from the general population to identify those who have or are likely to have a disorder. A secondary level screening is where a child is at risk for a developmental disorder but for whom a differential diagnosis has not yet been made [21].

Furthermore, usefulness of a screening test is contingent upon indices such as sensitivity, specificity, and predictive ability. Sensitivity is the accuracy of the test in identifying the disorder. Specificity is the accuracy of the test in identifying individuals who are not disordered. Predictive ability is the proportion of individuals with positive screening results who are confirmed by diagnostic tests as disordered. It is important to have a higher sensitivity (i.e., should be no less than $80 \%)$ than a higher specificity [21].

A review of available screening tests for ASD indicates several measures that meet the time restriction and psychometric measures. These screening tests are included in Table 1 . Six tests met psychometric measures needed for a screening instrument. These are the Modified-Checklist for Autism in Toddlers (M-CHAT) [22], Modified Checklist for Autism Toddlers-Revised with Follow-up (MCHAT-R/F) [23], Screening Tool for Autism in Two-Year-Olds (STAT) [24,25], Pervasive Developmental Disorder Screening Test-II (PDD-ST) [26], Chinese Autism Screening Test (CAST) [27], and Social Communication Questionnaire (SCQ) [28]. Three of the tests 
are appropriate for younger children under age two-years (i.e., $\mathrm{M}$ CHAT, M-CHAT-R/F, and CAST), two were appropriate for children between two- and three-years of age (i.e., STAT, PDD-ST) and one test was for children four-years and older (i.e., SCQ). One of the tests screened older children (i.e., CAST). Furthermore, all six of the tests were Stage-2 instruments with three consisting of questionnaire formats (i.e., M-CHAT, M-CHAT-R/F, SCQ), two observational formats (i.e., STAT, PDD-ST), and one consisting of both questionnaire and observational formats (i.e., CAST). The two MCHAT questionnaire instruments reported guidelines of 5-minutes whereas the remainder reported times of 10 - to 20 minutes to administer. All six of the tests reported high sensitivity measures and all, but one, indicated high specificity scores (i.e., the CAST did report a moderate level of specificity). Positive predictive ability was good for four of the tests (i.e., M-CHAT-R/F, STAT, CAST, and SCQ), while one was low (i.e., M-CHAT), and one did not report positive predictive ability (i.e., PDD-ST). Thus, several available screening tests are age appropriate, quick to administer, and appear to predict individuals with ASD with high accuracy [28].

\begin{tabular}{|c|c|c|c|c|}
\hline $\begin{array}{l}\text { Screening } \\
\text { tests }\end{array}$ & Ages & Format & Description & $\begin{array}{l}\text { Sensitivity /Specificity / } \\
\text { +Predictive }\end{array}$ \\
\hline М-CHAT ${ }^{a}$ & $\begin{array}{l}16-30 \\
\text { month } \\
\mathrm{s}\end{array}$ & $\begin{array}{l}\text { 2-Stage } \\
\text { Questionnaire }\end{array}$ & $\begin{array}{l}20 \\
\text { Questions } \\
<5 \text { minutes }\end{array}$ & $\begin{array}{l}\text { Sen=0.91(high) } \\
\text { Spec }=0.96 \text { (high) } \\
+ \text { Pred }=0.14(\text { low })\end{array}$ \\
\hline $\begin{array}{l}\text { M-CHAT- } \\
\text { R/F }\end{array}$ & $\begin{array}{l}16-30 \\
\text { month } \\
\mathrm{s}\end{array}$ & $\begin{array}{l}\text { 2-Stage } \\
\text { Questionnaire }\end{array}$ & $\begin{array}{l}20 \\
\text { Questions } \\
<5 \text { minutes } \\
\text { Follow-up } \\
\text { questions } \\
5-10 \\
\text { minutes }\end{array}$ & $\begin{array}{l}\text { Sen }=0.67 \text { (moderate) } \\
\text { Spec }=0.99 \text { (high)+Pred } \\
=0.51(\text { good })\end{array}$ \\
\hline STAT $^{\mathrm{C}}$ & $\begin{array}{l}24-36 \\
\text { month } \\
\mathrm{s}\end{array}$ & $\begin{array}{l}\text { 2-Stage } \\
\text { Observation }\end{array}$ & $\begin{array}{l}12 \text { items } 20 \\
\text { minutes }\end{array}$ & $\begin{array}{l}\text { Sen }=0.67 \text { (moderate) } \\
\text { Spec }=0.99 \text { (high) } \\
+ \text { Pred }=0.51 \text { (good) }\end{array}$ \\
\hline PDD-ST ${ }^{d}$ & $\begin{array}{l}24-35 \\
\text { month } \\
\mathrm{s}\end{array}$ & $\begin{array}{l}\text { 2-Stage } \\
\text { Observation }\end{array}$ & $\begin{array}{l}22 \text { items } \\
10-20 \\
\text { minutes }\end{array}$ & $\begin{array}{l}\text { Sen=0.92(high) } \\
\text { Spec=0.91(high) } \\
+ \text { Pred=(unknown) }\end{array}$ \\
\hline CAST $^{\mathrm{e}}$ & $\begin{array}{l}12-84 \\
\text { month } \\
\mathrm{s}\end{array}$ & $\begin{array}{l}\text { 2-Stage } \\
\text { Observation } \\
\text { and } \\
\text { Questionnaire }\end{array}$ & $\begin{array}{l}20 \text { items } \\
>10 \text { minutes }\end{array}$ & $\begin{array}{l}\text { Sen }=0.92 \text { (high) } \\
\text { Spec }=0.42 \text { (moderate) } \\
+ \text { Pred }=0.75 \text { (good })\end{array}$ \\
\hline$S_{C} Q^{f}$ & $\begin{array}{l}4 \\
\text { years }\end{array}$ & $\begin{array}{l}\text { 2-Stage } \\
\text { Questionnaire }\end{array}$ & $\begin{array}{l}40 \text { items } 10 \\
\text { minutes }\end{array}$ & $\begin{array}{l}\text { Sen }=0.96 \text { (high) } \\
\text { Spec }=0.80 \text { (high) } \\
+ \text { Pred }=0.93 \text { (good) }\end{array}$ \\
\hline
\end{tabular}

Table 1: Screening Instruments for determining ASD in children. (A)Modified Checklist for Autism in Toddlers-Revised. (b) Modified Checklist for Autism in Toddlers-Revised with Follow-up. (c) Screening Tool for Autism in Toddlers \& Young Children. (d) Pervasive Developmental Disorder Screening Test-II. (e) Chinese Autism Screening Test. (f) Social Communication Questionnaire

\section{Evaluation Protocol}

A diagnosis is different from an evaluation for a child with ASD. A diagnosis uses the evaluation results to make a decision as to whether the child has ASD or some other impairment. Below is a typical list of areas that need to be followed when completing an evaluation. Each area will be discussed briefly and will include diagnostic instruments that are commonly used to evaluate the areas.

\section{Hearing}

A child needs to have a hearing evaluation to rule out any concerns that may be present if the child does not respond. A hearing evaluation includes pure-tone conduction to measure the transmission of the sound through the outer ear, and a bone conduction evaluation to measure the sensory organ (i.e., cochlea). Typically, these measures are completed in a sound-treated booth by an audiologist.

\section{Cognition-intellectual functions}

Standardized testing of intelligence is for determining the child's Verbal IQ (VIQ), Performance IQ (PIQ), and Full Scale IQ (FSIQ). Two common tests for children are the Wechsler Intelligence Scale for Children-IV (WISC-IV) [29] and the Wechsler Preschool and Primary Scale of Intelligence (WPPSI-III) [30]. Typically, a clinical psychologist administers the WISC-IV or WPPSI-III. There are other tests that psychologists use besides the two WISCs. One of these is the Wechsler Individual Achievement Test-III [31], an individually administered instrument designed to estimate current levels of reading, mathematics, and written language. The WIAT-III provides composite scores that are derived from the administration of certain combinations of subtests. In addition, they may administer the NEPSY-A Developmental Neuropsychological Assessment [32] for evaluating processing skills. The NEPSY subtests are attention and executive functions, cognitive processes underlying language production, visuospatial abilities, sensorimotor integration, and memory and learning processes. Furthermore, the psychologist may have the parent complete the Behavior Assessment System for Children-Second Edition (BASC-2) [33]. The BASC-2 is a behavior rating scale that provides information on how behavior can affect academic performance. Vineland Adaptive Behavior Scale-II (Vineland-II) [34] is another critical evaluation scale in diagnosing and classifying ASD. There are five domain tests in the scale: Communication, Daily Living, Socialization, Motor Skills Domain and an optional Maladaptive Behavior Index. The age range encompasses birth to age 90 (Survey Interview, Expanded Interview, Parent/ Caregiver Rating Form) and 3 to 21-11 (Teacher Rating Form).

\section{IQ Comorbidity}

ASD has been linked to intellectual impairment with nearly $70 \%$ of individuals with ASD scoring below-average IQ [35]. It has also been recommended [20] that a child with a Full Scale score greater than IQ points 70 should be considered mild ASD, a child with a Full Scale score between IQ points 55-70 should be considered moderate ASD, and a child with a Full Scale score less than 50 IQ points should be considered severe ASD. Evidence from genetic studies [36-38] suggests that high versus low Verbal or Full Scale IQ may provide a subtyping of children with ASD. For example, Black, Wallace, Sokoloff, and Kenworthy [39] found individuals with ASD with higher Verbal IQ (VIQ) than Non-verbal IQ (NVIQ) demonstrated better adaptive communication skills but not socialization or Daily Living Skills. Similarly, Joseph et al. [35] found a dissociation between Verbal IQ and Non-verbal IQ between younger (i.e., 5;5 years) and older (i.e., $8 ; 11$ years). The greatest discrepancies (85\%) were when VIQ was lower than NVIQ. However, the older children with ASD differed greatly from the younger children. First, a VIQ score greater than NVIQ occurred in $28 \%$ of the older children but only $8 \%$ in the 
younger individuals. Second, the occurrence of VIQ scores being the same as NVIQ was about the same for both groups of children. Thirdly, asymmetry in the VIQ and NVIQ clusters differed between the two groups of children. That is, there were selective specializations and reorganization differences found in verbal and non-verbal skills for the older children but a dissimilar path of development occurred among the younger children. Joseph et al. [35] concluded that measuring IQ may be useful in determining not only cognitive strengths and weaknesses in children with ASD but also subtypes of autism. In a related study, Mayes and Calhoun [40] reported on lowand high-IQ groups of children with ASD on the Wechsler Intelligence Scale for Children-IV (WISC-IV) [29]. The investigators found that both groups performed well on Information, Similarities, and Vocabulary domains, indicating strengths in these areas. Scores on the Comprehension were lower than on Similarities, Vocabulary, and Information, indicating a relative weakness in verbal comprehension and social reasoning. Some patterns did differ between the two groups: for the low-IQ group, Block Design and Object Assembly were strengths, whereas in the high-IQ group, Block Design and Object were not strength but were weaknesses. The high-IQ group also demonstrated weaknesses on Coding and VMI subtests, indicating graph motor (writing) problems. The high-IQ group also performed lower on Digit Span and Arithmetic. Mayes and Calhoun [40] concluded that their findings have implications for educational intervention to assist in addressing appropriate educational settings.

\section{Oral-motor function tests}

Several tests measure non-verbal oral motor function, motor speech function, and oral structure. Two commonly used tests are Kaufman Speech Praxis Test for Children (KSPT) [41] and the Verbal Motor Production Assessment for Children (VMPAC) [42]. For a thorough review of these and other tests, see McCauley and Strand [16].

\section{ASD specific diagnostic tests}

It is difficult to label a child as ASD by a diagnostic test alone [43]. Experts recommend a supplement to the diagnostic test and viewing all the evaluation information before labeling a child. Standardized Tests for ASD are frequent and vary in length and concepts. Most are comprehensive and measure several dimensions related to the DSM-V broad categories. Three common tests include the Autism Diagnostic Observation Schedule-2 (ADOS-2) [43], Psychoeducational Profile-3rd (PEP-3) [44], and Autism Diagnostic Interview- Revised (ADI-R) [45]. One test, the Chinese Autism Diagnostic Scale (CADS) [20] is the first instrument designed, developed, and standardized in China for Chinese children with ASD. The CADS has seven major domains and two supplemental domains: Motor/Imitation behaviors, stereotyped behaviors, Sensory behaviors, Play behaviors, Social Interaction behaviors, Receptive Language skills, Expressive Language skills, and Supplemental domains for Academic skills and Executive Function skills.

\section{Speech-articulation}

A few evaluation instruments measure the specific speech items that a child produces in the initial, medial, and final position of words. In the English language format, the consonants are represented by pictures, and the child is asked to label the picture. An example of one such test is the Goldman-Fristoe Test of Articulation-2 (GFTA-2) [46]. Other measures of speech include speech intelligibility during discourse, and stimulability measures of error productions. One measure of speech intelligibility is using Percentage of Consonants Correct (PCC) [47]. Levels of intelligibility are determined from a 5minute speech sample. A stimulability test requires the child to imitate each consonant produced by the examiner in a Consonant+Vowel context, like "da", "sa", etc.

\section{Language tests}

There are four broad areas of assessment under the category of language. One is measuring vocabulary, another is measuring receptive language skills, the third is measuring expressive language skills, and the fourth measures pragmatics. Probably the most frequently used test for measuring vocabulary is the Peabody Picture Vocabulary Test-4th (PPVT-4) [48]. The child is presented four pictures and is asked to point to the correct item. It is norm referenced. Measuring receptive and expressive language in young children often requires pictures and objects. One such instrument, Preschool Language Scales, Fifth Edition (PLS-5) [49], contains items from pre-verbal interaction skills to emerging language. It is appropriate for children from birth through age 7 years. Other preschool language tests include the Test of Early Language Development-3 (TELD-3) [50], Clinical Evaluation of Language Fundamentals-Preschool, Second Edition (CELF-P) [51], and the Sequence Inventory of communication Development-Revised (SICDR) [52]. Testing instruments for pragmatic language are typically designed for older children. One instrument measuring pragmatics is the Test of Pragmatic Language-Second Edition (TOPL-2) [61]. it is for older children ages 6 years to 19 years and assists in identifying pragmatic language deficits.

\section{Academic and literacy tests}

Commonly the areas of academics are divided between reading skills and math skills. Reading encompasses many areas, including knowing the alphabet, knowing the sounds of letters, phonemic awareness (e.g., sound blending, changing first sounds in a CVC word, rhyme, etc.), emergent literacy (e.g., knowing a word is a word, reading from front-to-back, identifying community signs, where to begin to read in a sentence, etc.), reading sight words, and reading for comprehension. For a good review of Issues in emergent literacy, refer to the article written by Kaderavek and Sulzby [53].

Math is also comprehensive and includes such areas as counting; adding and subtracting; word problem solving; concepts of none, more, less, most, smaller, smallest, bigger, biggest; names of common shapes and recognize those shapes in the child's environment; sorting by size, shape, and color; following simple verbal math directions such as, "show me the one red square," or "Take away two blue crayons." An online computer assessment program is available through STAR [54].

\section{Play skills assessment}

Children with ASD have been found to spend less time in functional and symbolic play than with their peers [55]. Most professionals recommend using an observational scale without norms to describe a child's play behavior. For instance, the Play Assessment Scale (PAS) [56] contains 45 items that are developmentally sequenced. Another approach, developed by Linder [57] called Trans disciplinary Play-Based Assessment (TPBA), requires a team approach consisting of a play facilitator who engages the child, a parent facilitator who is responsible for discussing the process, a person to 
operate a video camera, and other professionals. Linder has a detailed coding guideline for cognitive, social-emotional, communication and language, and sensorimotor development. Another approach, Symbolic Play Scale developed by Westby [58] incorporates a relationship between symbolic play, activities, and language. It goes from 18 months to 6 years of age.

\section{Sensory motor impairment assessment}

Due to the nature, that many children with ASD also have sensory motor impairment, tests for sensory profile and visual motor integration scales are often administered. The Sensory Profile [59] is a 125-question profile for caregivers, for the frequency with which their child responds to various sensory experiences. The profile provides a comprehensive picture of a child's sensory processing pattern. The Sensory Profile contains a 38-item caregiver questionnaire and score sheet designed for use in screening and evaluating research protocols. The items on the Sensory Profile are grouped into three major sections: sensory processing, modulation, and behavioral and emotional responses. The age range varies anywhere from 3-10 or 5-10 years old. Beery-Buktenica Developmental Test of Visual-Motor Integration (Beery VMI) [60] assesses the extent to which individuals can integrate their visual and motor abilities. The test presents the examinee with drawings of 24 geometric forms, arranged in developmental sequence, from less to more complex. The test can be individually or group administered in just 10 to 15 minutes. The age ranges vary from two through 18 years old. A Short Form composed of 15 drawings is often used with 2- to 8-year-old children. VMI also has two supplemental tests--the VMI Visual Test and the VMI Motor Test. Both supplemental tests can be administered in 5 minutes or less. Generally, they are administered if full- or short-form VMI results indicate a need for further testing.

\section{Social impairments}

Social communication and social interaction includes such failures as back-and-forth conversation, reduced sharing of interests, emotions, or affect and failure to initiate or respond to social interactions [1]. It also may include deficits in pragmatic skills such as poor eye contact, joint attention, failure to understand gestures, and facial expressions. Social deficits includes failure in developing, maintaining, and understanding relationships, such as, difficulties in adjusting to social contexts, difficulties in imaginative play or making friends, and absence of peers. Assessing social impairments and interactions are, therefore, important areas to measure.

The Social Responsive Scale-Second Edition (SRS-2) [60] is a frequently used test for assessing social skills in children with ASD. The SRS-2 assesses various dimensions of interpersonal behavior, communication, and repetitive/stereotypic behavior often associated with ASD. The test includes four different forms, each with 65 items, across different age groups: preschool (ages 29 months to 53 months), school-age (4 to 18 years), adult form (ages 19 and up), and Adult SelfReport (ages 19 and up). It takes approximately 15-20 minutes to administer. Specificity has been reported to be moderate (i.e., 78), sensitivity is high (i.e., 94) and positive predictive value is good (i.e., . 98). More than 40 research investigations have documented the diagnostic validity of the SRS-2. There is evidence of good inter-rater reliability, high internal consistency, and convergent validity with the Autism Diagnostic Interview-Revised (ADI-R) [45], Autism Diagnostic Observation Schedule (ADOS-2) [43], and Social Communication Questionnaire (SCQ) [28]. The two symptom domains for Social Communication and Restricted Interests and Repetitive Behaviors follow the approved DSM-5 [1] criteria for ASD.

\section{Summary}

Evaluation and diagnosis of children with ASD is as much a skill as it is a science. In this brief review, attempts have been made to provide the basics for doing both. It is strongly urged that individuals who are planning to serve children with ASD increase their experience working with these children, learn more about test administration, and especially learn about interpreting test results for accurate diagnoses and appropriate treatment strategies for the children.

It is the fervent belief that a team approach is necessary to fully assess and determine the level of autism; the strengths and weaknesses of the child's social, communication, and repetitive-stereotype behaviors; and appropriate planning of intervention to assist the child's needs. A lot has been written on children with ASD in general, but in truth, the individual child is unique and most often does not present with behaviors like other children. This uniqueness makes the decision for diagnosing the child most difficult and challenging. For this reason, a team of professionals is highly recommended.

Assessing the child's skills in language, play, and sensory motor can help the professional plan an individualized intervention program. Involving the parents, in the assessment, is also important for understanding the home routines; how the child functions socially; and how the child interacts with peers, family members, and teachers. A comprehensive assessment, therefore, provides a fuller understanding of the child and his needs.

\section{References}

1. Diagnostic and statistical manual of mental disorders (2013). (5th edn) (DSM-V), American psychiatric association, Arlington, VA.

2. Crais ER, Watson LR, Baranek GT, Reznick JS (2006) Early identification of autism: how early can we go? Semin Speech Lang 27: 143-160.

3. Tager-Flusberg $\mathrm{H}$ (2000) Language and understanding minds: connections in autism. In: Baron-Cohen S, Tager-Flusberg H, Cohen DJ (Eds) Understanding other minds: Perspectives from autism and developmental cognitive neuroscience. (2nd edn), Oxford University press, Oxford.

4. Smith V, Mirenda P, Zaidman-Zait A (2007) Predictors of expressive vocabulary growth in children with autism. J Speech Lang Hear Res 50: 149-160.

5. Chakrabarti S, Fombonne E (2005) Pervasive developmental disorders in preschool children: confirmation of high prevalence. Am J Psychiatry 162: 1133-1141.

6. Bzoch K, League R, Brown V (2003) Receptive-expressive emergent language test. (3rd edn), Proed, Austin, TX.

7. Rossetti L (2006) The Rossetti infant-toddler language scale. Lingui Systems, East Moline, IL.

8. Fenson L Marchman V, Thal D, Dale P, Reznick J, et al. (2006) The MacArthur-Bates communicative development inventories. Brookes Publishing, Baltimore, MD.

9. Baranek G, Watson L, Crais E, Reznick JS (2009) First Year Inventory, Lite (FYI-Lite) Chapel Hill, NC: University of North Carolina at Chapel Hill.

10. Reznick J, Barenek G, Shaye R, Watson L, Crais E (2007) A parent-report instrument for identifying one-year-olds at risk for an eventual diagnosis of autism: The first year inventory. J Autism Dev Disord 37: 1691-1709

11. Bryson S, McDermott C, Rombough V, Brian J, Zwaigenbaum L (2007) The autism observation scale for infants. Unpublished Scale, Toronto, ON. 
12. Bryson SE, Zwaigenbaum L, McDermott C, Rombough V, Brian J (2008) The autism observation scale for infants: scale development and reliability data. J Autism Dev Disord 38: 731-738.

13. Layton T, Hao G, Zou X, Li L (in press) Differentiating low and high functioning children with autism, children with intellectual disability, and children with typical development in a Chinese population J Intellect Dev Disabil.

14. Spencer PE (1996) The association between language and symbolic play at two years: evidence from deaf toddlers. Child Dev 67: 867-876.

15. Schreck KA, Williams K, Smith AF (2004) A comparison of eating behaviors between children with and without autism. J Autism Dev Disord 34: 433-438.

16. McCauley RJ, Strand EA (2008) A review of standardized tests of nonverbal oral and speech motor performance in children. Am J Speech Lang Pathol 17: 81-91.

17. Ayres AJ (1974) The development of sensory integrative theory and practice: A collection of the works of A. Jean Ayres. Kendall Hunt publishing company, Dubuque, IA.

18. Brown WT (2010) Genetics in autism. In: Chauhan A, Chauha V, Brown WT (Eds) Autism: Oxidative stress, inflammation, and immune abnormalities. Taylor and Francis group, Boca Raton, FL.

19. Hallmayer J, Cleveland S, Torres A, Phillips J, Cohen B, et al. (2011) Genetic heritability and shared environmental factors among twin pairs with autism. Arch Gen Psychiatry 68: 1095-1102.

20. Hao G, Layton TL, Zou XB, Li DY (2014) Evaluating autism in a Chinese population: the Chinese autism diagnostic scale. World J Pediatr 10: 160-163.

21. Sturner R, Layton T, Evans A, Heller J, Funk, et al. (1994) Preschool speech and language screening: A review of currently available tests. Am J Speech Lang Pathol 3: 25-36.

22. Robins D, Fein D, Barton M, Green J (2001) The modified checklist for autism in toddlers: an initial study investigating the early detection of autism and pervasive developmental disorders. J Autism Dev Disorder, 31: 131-144.

23. Robins DL, Casagrande K, Barton M, Chen CM, Dumont-Mathieu T, et al. (2014) Validation of the modified checklist for Autism in toddlers, revised with follow-up (M-CHAT-R/F). Pediatrics 133: 37-45.

24. Stone W, Ousley O (1997) STAT Manual: Screening tool for autism in two-year-olds. Unpublished manuscript, Vanderbilt University.

25. Stone WL, Coonrod EE, Ousley OY (2000) Brief report: screening tool for autism in two-year-olds (STAT): development and preliminary data. J Autism Dev Disord 30: 607-612.

26. Siegel B (1998) Pervasive developmental disorder screening test-II. Pearson Publishing, NY.

27. Hao G, Layton $\mathrm{T}$, Zou $\mathrm{X}$ (in press) Chinese autism screening test. People's medical publishing Services, Beijing, P.R. China..

28. Rutter M, Bailey A, Bedrument S, Lord C, Pickles A (2006) Social communication questionnaire. Western psychological services, Los Angeles, CA.

29. Wechsler D (2003) Wechsler intelligence scale for children-IV. Pearson publishing Group, San Antonio, TX.

30. Wechsler D (2002) Wechsler preschool and primary scale of intelligenceIII. Pearson publishing group, San Antonio, TX.

31. Wechsler D (2009) Wechsler individual achievement test-III. Pearson publishing group, San Antonio, TX.

32. Korkman M, Kirk, Kemp S (2007). NEPSY-A developmental neuropsychological assessment-II. Pearson publishing group, San Antonio, TX.

33. Reynolds C, Kamphaus R (2004) Behavior assessment system for children. (2nd edn), Pearson publishing group, San Antonio, TX.

34. Sparrow SS, Cicchetti, DC, Balla DA (2005) Vineland adaptive behavior scales. (2nd edn), (Vineland-II) Pearson publishing group, San Antonio, TX.
35. Joseph R, Tager-Flusberg H, Lord C (2002) Cognitive profiles and socialcommunicative functioning in children with autism spectrum disorder. Journal of Child Psychology and Psychiatry, 43: 807-821.

36. Bolton P, Macdonald H, Pickles A, Rios P, Goode S, et al. (1994) A casecontrol family history study of autism. J Child Psychol Psychiatry 35: 877-900.

37. Fein D, Stevens M, Dunn M, Waterhouse L, Allen D, et al. (1999). Subtypes of pervasive developmental disorder: Clinical characteristics. Child Neuropsychol 5: 1-23.

38. Szatmari P, MacLean J, Jones M, Bryson S, Zwaigenbaum L, et al. (2000) The familial aggregation of the lesser variant in biological and nonbiological relative of PDD probands: A family history study. J Child Psychol Psychiatry 41: 579-586.

39. Black DO, Wallace G, Sokoloff JL, Kenworthy L (2009) Brief report: IQ split predicts social symptoms and communication abilities in highfunctioning children with autism spectrum disorders. J Autism Dev Disord 39: 1613-1619.

40. Mayes SD, Calhoun SL (2003) Analysis of WISC-III, Stanford-Binet:IV, and academic achievement test scores in children with autism. J Autism Dev Disord 33: 329-341.

41. Kaufman N (1995) Kaufman speech praxis tests for children. Wayne State University press, Detroit, MI.

42. Hayden D, Square P (1999) Verbal motor production assessment for children. The psychological corporation, Austin, TX.

43. Lord C, Rutter M, Dilavore B, Risi P (2001) Autism diagnostic observation schedule. Western psychological Services, San Diego, CA.

44. Schopler E, Lansing M, Reichler R, Marcus L (2005) PEP-3: Psychoeducational profile. (3rd edn), Western Psychological Services, Los Angeles, CA.

45. Lecouteur A, Rutter M, Lord C (2003) Autism diagnostic interviewrevised (ADI-R). Evaluating children with ASD: 21. Pearson publishing group, San Antonio, TX.

46. Goldman R, Fristoe M (2000) Goldman-Fristoe test of articulation-2. Pearson publishing group. San Antonio, TX.

47. Shriberg LD, Austin D, Lewis BA, McSweeny JL, Wilson DL (1997) The percentage of consonants correct (PCC) metric: extensions and reliability data. J Speech Lang Hear Res 40: 708-722.

48. Dunn L, Dunn D (2007) Peabody picture vocabulary test. (4th edn), Pearson publishing group, San Antonio, TX.

49. Zimmerman I, Steiner V, Pond R (2002) Preschool language scale. (5th edn), Psychological Corporation, New York.

50. Hresko W, Reid DK, Hammill D (1991) Test of early language development. (3rd edn), Pearson Publishing Group, San Antonio, TX.

51. Wiig EH, Secord W, Semel EM (2004) Clinical evaluation of language fundamentals-preschool. (2nd edn), Psychological Corporation, New York.

52. Hendrick D, Prather E, Tobin A (1984) Sequenced inventory of communication development-revised. Proed, Austin, TX.

53. Kaderavek J, Sulzby E (1999) Issues in emergent literacy for children with language impairments. CIERA/University of Michigan.

54. Enterprise assessments-STAR Reading and STAR Math at http:// www.renaissance.com/products/star-assessments/star-reading

55. Jarrold C, Boucher J, Smith P (1996) Generativity deficits in pretend play in autism. Brit J Dev Psychol 14: 275-300.

56. Fewell RR., Rich JS (1987) Play assessment as a procedure for examining cognitive, communication, and social skills in multi-handicapped children. J Psychoeduc Assess 2: 107-118.

57. Linder TW (1993) Trandisciplinary play-based assessment: A functional approach to working with young children. (2ndedn), Paul Brookes, Baltimore, MD.

58. Patterson J, Westby C (1998) The development of play. In: Shulman B, Haynes HO (Eds) Communication development. (2nd edn), Englewood Cliffs, NJ.

59. Dunn W (1999) Sensory profile. Pearson publishing group, San Antonio, TX. 
Citation: Layton T, Hao G (2014) Early Assessment in Autism Spectrum Disorders . J Psychol Abnorm Child 3: 1000130. doi: 10.4172/2329-9525.1000130

Page 7 of 7

60. Constantino JN (2012) Social responsiveness scale. (2nd edn), Western Psychological Services, Los Angeles, CA.
61. Phelps-Terasaki D, Phelps-Gunn T (2007) Test of Pragmatic LanguageSecond. Proed, Austin, TX. 